myjournal manuscript No.

(will be inserted by the editor)

\title{
Efficient $k$-edge Connected Component Detection through an Early Merging and Splitting Strategy
}

\author{
Heli Sun • Jianbin Huang • Yang Bai . \\ Zhongmeng Zhao • Xiaolin Jia • Fang \\ He . Yang Li
}

Received: date / Accepted: date

\begin{abstract}
Computing $k$-edge connected components can be used to capture closely related vertices in a graph. It is an important problem with many applications. Due to the high time complexities of traditional algorithms for computing $k$-edge connected components, it is difficult for them to be applied to efficiently process large scale graphs. In this paper, an early merging and splitting based maximal $k$-edge connected subgraph detection algorithm, named MSK, is proposed. It computes the $k$-edge connected components of a graph with an order list of vertices which is decided according to the connectivity of vertices in the graph. During the processing of the vertex list, if two vertices are $k$-edge connected, they are merged into a super-vertex. On the contrary, if the vertex pair does not satisfy the condition of $k$-edge connectivity, the related vertices and edges are removed from the graph, and the
\end{abstract}

Heli Sun

Department of Computer Science and Technology, Xi'an Jiaotong University, Xi'an 710049, China

Jianbin Huang

School of Software, Xidian University, Xi'an, 710071, China

Yang Bai

School of Software, Xidian University, Xi'an, 710071, China

Zhongmeng Zhao

Department of Computer Science and Technology, Xi'an Jiaotong University, Xi'an 710049, China

Xiaolin Jia

Department of Computer Science and Technology, Xi'an Jiaotong University, Xi'an 710049, China

Fang $\mathrm{He}$

Department of Computer Science and Technology, Xi'an Jiaotong University, Xi'an 710049, China

Yang Li

Department of Computer Science and Technology, Xi'an Jiaotong University, Xi'an 710049, China 


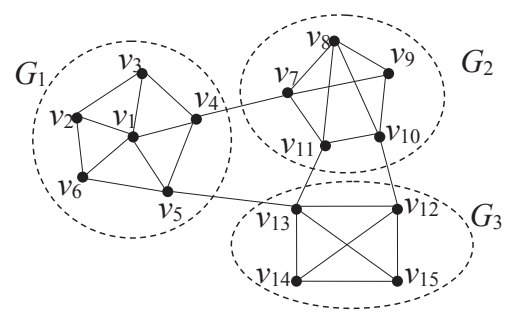

Fig. 1: A toy graph with three 3-edge connected subgraphs

graph is decomposed into several subgraphs. The above steps are performed iteratively in the obtained subgraphs until each subgraph become a $k$-edge connected component. In order to further improve the time efficiency, an approximate algorithm PMSK is also proposed. The experimental results on a large number of real and synthetic graphs show that the proposed algorithms are accurate and more efficient than the state-of-the-art algorithms.

Keywords Graph decomposition · Subgraph · $k$-edge connectivity · Graph mining

\section{Introduction}

With the emergence of applications in social networks, biological networks, communication networks, and the World Wide Web, various complex data can be represented by graphs. The problem of discovering $k$-edge connected components or maximal $k$-edge connected subgraphs in these graphs is a challenging problem [1]. Given a graph $G=(V, E)$, where $V$ is the vertex set and $E$ is the edge set, a graph $G$ is $k$-edge connected indicated that the remaining graph is still connected after the removal of any $k-1$ edges from $G$. The problem of computing $k$-edge connected components of $G$ is to find a maximal set of subgraphs of $G$ that are $k$-edge connected. As shown in Fig.1, the graph $G$ is a 2-edge connected graph, and $G_{1}, G_{2}$, and $G_{3}$ are its three 3-edge connected subgraphs. Computing $k$-edge connected components can be used in many real applications $[2,3,4,14]$. For example, the closely related relationships or communities can be identified by using computing $k$-edge connected components from social networks [5]. A group of highly connected web pages can be discovered in a web-link based graph which can provide useful information for web page searching. In biological networks, biologists can use $k$-edge connected subgraphs technology to detect highly connected gene data.

If graph $G$ is not $k$-edge connected, there must be a set $C$ of edges such that the number of edges $|C|<k$, the removal of the edges in set $C$ cuts the graph into at least two disconnected subgraphs $G_{1}$ and $G_{2}$. Based on this, a minimum cut based algorithm MinimumCut was proposed in [7] to iteratively cut a non $k$-edge connected graph into two disconnected subgraphs until all 
remaining connected subgraphs are $k$-edge connected or no edge is remaining in the graph. For example, considering the graph in Fig.1, let $k=3$, it is easy to get that the minimum cut is $\left\{\left\{v_{4}, v_{7}\right\},\left\{v_{5}, v_{13}\right\}\right\}$, the cut value is less than $k$. After the removal of edges $\left\{v_{4}, v_{7}\right\}$ and $\left\{v_{5}, v_{13}\right\}$, the graph is decomposed into two disconnected subgraphs $G_{1}$ and $G_{2} \cup G_{3}$, where $G_{1}$ contains vertices $v_{1}, v_{2}, v_{3}, v_{4}, v_{5}$, and $v_{6}$, and $G_{2} \cup G_{3}$ contains vertices $v_{7}, v_{8}, v_{9}, v_{10}, v_{11}, v_{12}$, $v_{13}, v_{14}$, and $v_{15}$. In the next, the minimum cut based algorithm is executed against subgraphs $G_{1}$ and $G_{2} \cup G_{3}$, respectively. Since the minimum cut value of subgraph $G_{1}$ is 3 (i.e., $k=3$ ), the subgraph $G_{1}$ is 3-edge connected subgraph. The minimum cut of $G_{2} \cup G_{3}$ is $\left\{\left\{v_{10}, v_{12}\right\},\left\{v_{11}, v_{13}\right\}\right\}$ and the corresponding cut value is 2 . After the removal of the edges in this cut, the subgraph $G_{2} \cup G_{3}$ is decomposed into two subgraphs $G_{2}$ and $G_{3}$. Since the minimum cut value of subgraphs $G_{2}$ and $G_{3}$ are 3, the iteration is terminated and all the 3-edge connected subgraphs $G_{1}, G_{2}$ and $G_{3}$ are obtained. In [8] and [9], the authors also presented minimum cut based algorithms to compute $k$-edge connected components. However, the main weakness of the minimum cut based algorithms are their low time efficiency. Recently, a graph decomposition based algorithm DecB-LMSD is proposed to reduce the running time of computing $k$-edge connected components [1]. In the process of DecB-LMSD, the vertices are ordered in each iteration, some vertices are merged into super-vertices during the processing of ordering. At the end, each super-vertex corresponds to a $k$-edge connected subgraph in the original graph. Since the method may result in the deviation of the result, it can only be considered as an approximate algorithm.

In this paper, we propose an early merging and early splitting based exact algorithm MSK to compute $k$-edge connected components. The proposed method first computes an order list $L$ of vertices based on the connectivity of vertices in a graph. During the computing of the order list $L$, the early merging and splitting strategies are performed. If two vertices are $k$-edge connected, they are merged into a super-vertex. On the contrary, if the vertices do not satisfy the condition of $k$-edge connectivity, the related vertices and edges are removed from the graph, and the graph is decomposed into several subgraphs. The above steps are performed iteratively until each subgraph is a $k$-edge connected component. It has to be noted that the graph splitting will result in some $k$-edge connected vertices in the original graph do not possess the $k$-edge connectivity in the subgraphs. Therefore, in order to guarantee the correctness of the result, the super-vertex within a subgraph is restored as the original state before the starting of the next iteration. Furthermore, the proposed MSK algorithm does not need to compute the minimum cut at each iteration. In order to improve the time efficiency, we also propose an approximate algorithm PMSK in this paper.

The main contributions of the paper can be summarized as follows.

(1) We proposed an exact algorithm MSK to detect the k-edge connected components. The algorithm is based on the early merging and splitting strategy, and its time complexity is $O(h \times|E|)$, where $h$ is the number of iterations and $|E|$ is the number of edges. 
(2) In order to further improve the time efficiency, an approximate algorithm PMSK is proposed to compute the $k$-edge connected components.

(3) The extensive experimental results show that the proposed methods are accurate and are more efficient than the state-of-the-art methods.

The remaining of the paper is organized as follows. The related work is discussed in section 2. Section 3 provides the preliminary information including the concept definition. Section 4 presents the proposed $k$-edge connected subgraph algorithms. The experimental results are reported in section 6 . Finally, a conclusion is given in section 7 .

\section{Related work}

Since graph can be used to express the relationships of different objects for a wide range of applications, it has recently drawn a great deal of attentions. A large number of researches have been done on the design of subgraph computation algorithms [21,22].

Due to the importance of computing $k$-edge connected subgraphs in a graph, a lot of researches have been done. David R. karger [6] proposed a max-flow based graph decomposition algorithm [16], which computes maxflow based on random sampling. Then, it finds the minimum cut of the graph until all the obtained subgraphs are $k$-edge connected subgraphs. The complexity is $O(\operatorname{lm} \sqrt{n}), l$ is the number of iterations. The $k$-core of a graph can also be used to find $k$-edge connected subgraphs $[2,11,12]$ and [13]. It computes the degree of each vertex in a graph, and the vertices and related edges are removed from the graph when their degrees are less than a predefined value $k$. The degrees of vertices within all the obtained $k$-cores are more than $k$. P. Dankelmann [17] applied a concept of vertex connectivity to compute $k$-edge connected subgraphs. However, this method needs to preprocess a graph and the computation time is not stable. Takuya et al. present a nearly linear algorithm MkECSs [19]. It first removes vertices with degree less than $k$ and the relevant edges to get $k$-core. Then, the adjacent two vertices are selected randomly to be merged into a vertex. The result was used as the input to iteratively perform merging operation until the graph is empty. In order to improve the time efficiency, it randomly selects two adjacent vertices to be merges into a vertex. This will result in the results of $k$-edge connected subgraphs are uncertainty, e.g., when we use MkECSs algorithm to compute 3-edge connected subgraphs in Fig.1, since this graph is already 3-core, if we select adjacent vertices $v_{11}$ and $v_{13}$ to be merged into a vertex, then $v_{11}$ and $v_{13}$ will be contained in a single 3 -edge connected subgraph, which is inconsistent with the truth. Therefore, the result of MkECSs algorithm is uncertainty. [20] proposed a method to decompose a graph into maximal $k$-edge connected subgraphs based on random contraction of edges. It enumerated MkECSs, and it is several orders of magnitude faster than the previous method. However, the problems of $k$-cores based algorithms are that they only take account of the connectivity among the vertices of the subgraph and ignore the connectivity 
of the entire graph itself. And the computation of $k$-edge connected subgraphs emphasizes the connectivity of subgraphs rather than the connectivity of the global graph.

The minimum cut [7] based algorithms were proposed to compute $k$-edge connected components in [8], [10] and [18]. The cut-based framework plugged in the Testing Connectivity algorithm (CutB-TC) for computing $k$-edge connected components is to iteratively compute the minimum cut of edges, the edges with cut values less than $k$ are deleted from the graph, if the minimum cut values of all the subgraphs are larger than $k$, then the final $k$-edge connected subgraphs are found. However, the time complexity of computing minimum is high expensive, is $O\left(n m+n^{2} \log m\right)$, where $n$ is the number of vertices and $m$ is the number of edges. To tackle the problem, [9] proposed three speed-up techniques: vertex reduction, edge reduction and cut pruning to be applied on the minimum cut based algorithms to find the maximal $k$-edge connected subgraphs from a large graph. In order to improve the time efficiency, the algorithm DecB-LMSD [1] was proposed to reduce the running time of computing $k$-edge connected components. Although the time complexity was reduced to $O(h \times l \times|E|)$, it can only be considered as an approximate algorithm. Therefore, in this paper we focus on developing an accurate algorithm for computing $k$-edge connected components with low time complexity.

\section{Preliminary}

In this section, we first introduce some basic notations to address the computation of the $k$-edge connected components. Then we give the problem definition and the computation framework used in our method.

\subsection{Basic concepts}

In this paper, our work focus on an undirected graph. Let $G=(V, E)$ be an undirected graph, where $V$ is the set of vertices and $E$ is the set of edges. In this section, we introduce some basic notions which can be found in the literatures $[1,7,9]$.

Definition 1 (Vertex Induced Subgraph) Given a graph $G=(V, E), S$ denotes the subset of vertex set $V, G[S]=(S, E[S])$ is a sub-graph induced by vertex set $S$, where $E[S]=\{\{u, v\} \in E \mid u, v \in S\}$.

Definition 2 (Cut) Given a graph $G=(V, E)$, a cut $C=(S, T)$ is a partition of $V$ into two nonempty subsets $S$ and $T$, where $S \cup T=V$ and $S \cap T=\emptyset$.

We also denote a cut by the set of edges whose endpoints are in two different vertex subsets $S$ and $T$ respectively, i.e. $E[S, T]=\{\{u, v\} \in E \mid u \in S, v \in T\}$. The value of a cut $C$, denoted by $w(C)$, is the number of edges in the cut, i.e., $w(C)=|E[S, T]|=|\{\{u, v\} \in E \mid u \in S, v \in T\}|$. For example, in Fig.1, 
the edge $\left\{v_{4}, v_{7}\right\}$ and $\left\{v_{5}, v_{13}\right\}$ can be considered as a cut between subgraphs $G_{1}$ and $G_{2} \cup G_{3}$, and its cut value is 2 .

Definition 3 ( $s-t$ Cut) A cut $C=(S, T)$ is called an $s-t$ cut if two different vertices $s$ and $t$ are lie in two different components $S$ and $T$ respectively, where $s \in S$ and $t \in T$.

Definition 4 (Minimum Cut) The cut with minimum value in a graph $G$ is called a minimum cut. The value of the minimum cut of a graph $G$ is denoted by $\lambda(G)$. In the same way, we use $\lambda(s, t ; G)$ to denote the value of the minimum $s-t$ cut of $G$.

If the cut with value less than $k$, the graph is partitioned by the cut. Then we find the min-cut in the obtained subgraphs, the algorithm is end until all cuts of $G$ with values are not less than $k$. The general algorithm for computing minimum cut of a graph is MinimumCut [7]. This method is used to compute the global minimum cut of a graph. The main idea is finding minimum $s-t$ cuts for $(|V|-1)$ pairs of vertices, and reporting the global minimum cut with the smallest value. In order to compute a minimum $s-t$ cut, a maximum adjacency search (Mas) procedure is adopted, the vertices $s$ and $t$ are determined at the procedure Mas.

The procedure Mas can be described as computing an order list $L$ of all vertices in graph $G$. Let $t$ be the last vertex in $L$ and $s$ be the vertex prior to $t$. The adjacent edges of $t$ in $G$ is the minimum $s-t$ cut. The list $L$ is constructed as follows: initially, an arbitrary vertex in graph $G$ is selected to insert into the list $L$. Then, the vertex $u$ which is not included in $L$ and the one most tightly connected to $G\left[V_{L}\right]$, i.e., $u=\arg \max _{v \in V \backslash L} w(L, v)$, is added to the tail of $L$, where $w(L, v)$ denotes the number of edges between $v$ and the vertices in $L$.

\subsection{Problem statement}

In this paper, we handle the problem of efficiently detecting the $k$-edge connected components of the given graph $G[1,9,20]$. The related concepts are introduced as follows.

Definition 5 ( $k$-edge connected graph) A graph $G$ is $k$-edge connected if the remaining graph is still connected after the removal of any $(k-1)$ edges from $G$.

For example, the graph $G$ shown in Fig.1 is a 2-edge connected graph and the subgraphs $G_{1}, G_{2}$ and $G_{3}$ are 3-edge connected subgraphs.

Definition 6 ( $k$-edge connectivity between vertices $s$ and $t$ ) Two vertices $s$ and $t$ are called $k$-edge connected in $G$, if and only if the value of a minimum $s-t$ cut satisfies $\lambda(s, t ; G) \geq k$. 
Definition 7 ( $k$-edge connected component) Given a graph $G$, if a subgraph $G_{1}$ of $G$ is a $k$-edge connected graph and any super-graph of $G_{1}$ is not a $k$-edge connected graph, then $G_{1}$ is called a $k$-edge connected component of $G$.

A $k$-edge connected component of a graph is a maximal $k$-edge connected subgraph of it, and all $k$-edge connected components of a graph are disjoint [1]. Given an undirected graph $G$ and a parameter $k$, our goal is efficiently detecting all the $k$-edge connected components of $G$.

\subsection{Existing computation framework}

In [1], the authors present a graph decomposition-based $k$-edge connected component detection method DecB-LMSD. The algorithm DecB-LMSD was proposed to reduce the running time of computing $k$-edge connected components by using some optimization techniques. Given a graph $G$ and an integer $k$, it iteratively decomposes a non $k$-connected subgraph into several connected subgraphs by removing edges in all cuts of $G$ with values less than $k$. At the same time, whenever a minimum $s-t$ cut is found by Mas, $s$ and $t$ are merged into a super-vertex, and the resulting graph is given as an input to Mas for another iteration. Also, it can merge more than one pair of vertices during one iteration of Mas by using the early merging method, because the only requirement for merging is that the connectivity between $s$ and $t$ should be no less than $k$, as long as each pair of vertices is guaranteed to be $k$-connected in the input graph. Eventually, each super-vertex corresponds to a $k$-edge connected subgraph in the original graph.

However, we have found that the early merging method used in DecBLMSD may result in deviations of the result. As shown in Fig.2, let $k=3$, the graph $G$ consists of 3 -edge connected subgraphs are $G_{1}, G_{2}$ and $G_{3}$. However, after the first iteration of DecB-LMSD, the result of the vertices order is $v_{1}, v_{2}$, $v_{3}, v_{4,5}, v_{6}, v_{7}, v_{8}, v_{9,10}, v_{11}, v_{12,13,14}, v_{15}, v_{16,17}$, in which $v_{4,5}, v_{9,10}, v_{12,13,14}$, and $v_{16,17}$ are super-vertices formed by vertices $v_{4}, v_{5}, v_{9}, v_{10}, v_{12}, v_{13}, v_{14}$, and $v_{16}, v_{17}$. Since the super-vertices are not decomposed in the following iteration, the final 3-edge connected subgraphs are $G_{1}$ and $G_{2} \cup G_{3}$. However, the vertices $v_{4}$ and $v_{5}$ are not $k$-edge connected if we confirm vertices $v_{2}$ and $v_{3}$ are not $k$-edge connected and remove them from the graph $G$. Therefore, the DecBLMSD algorithm can only be considered as an approximate algorithm.

Our method use the similar computation framework with that in DecBLMSD. In order to guarantee the correctness of the result, we restore the super vertex within a subgraph before the next iteration. Furthermore, the minimum cut value does not need to be computed at each iteration, and the $k$-edge connected subgraphs can be computed during once minimum cut value computation. 


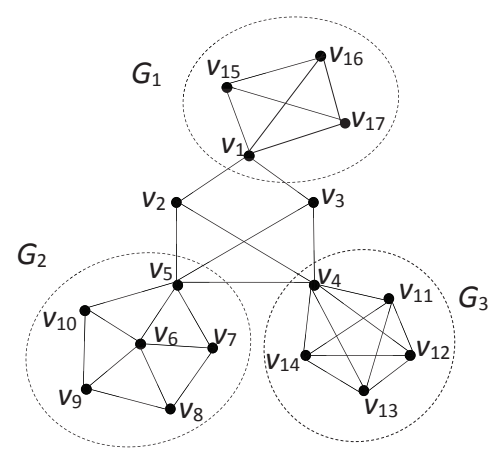

Fig. 2: The 3-edge connected components of a sample graph $\tilde{G}$

\section{The proposed exact algorithm MSK}

Given a graph $G=(V, E)$ and an integer $k$, the proposed method for computing $k$-edge connected components is iteratively computing the minimum cut value of the graph and decomposing it into several subgraphs by removing edges with values less than $k$. This procedure is continued until all the minimum cut values of the subgraphs are not less than $k$. The algorithms terminates when all subgraphs are $k$-edge connected. The procedure of graph decomposition can be organized into a tree structure. The graph $G$ is represented by the root vertex, each $k$-edge connected subgraph of $G$ is represented by a leaf vertex, and the intermediate vertices of the tree are subgraphs of $G$ that are not $k$-edge connected.

\subsection{An early merging and splitting strategy}

During the process of computing $k$-edge connected components, we apply vertex merging and subgraph splitting to improve the efficiency. We use $P G$ to indicate the set of subgraphs generated by merging or splitting for the process of next iteration. The proposed method adopts the procedure Mas to compute the minimum cut value. During the process of Mas, a linear order $L$ for the vertices of $G$ is generated. Assume that $u$ is the last vertex of $L$, for vertex $v$, if $w(L, v) \geq k$, then $\lambda\left(u, v ; G\left[V_{L} \cup v\right]\right) \geq k$. Therefore, we can say that vertices $u$ and $v$ are $k$-edge connected in subgraph $G\left[V_{L} \cup v\right]$. Then two vertices $u$ and $v$ are merged into a single super-vertex, the new super-vertex is denoted as $S_{u, v}$, the new generated graph is $\rho_{u, v}(G)$. The vertices $u, v$ and related edges are deleted from graph $G$ and the super-vertex $S_{u, v}$ is added to graph $G$. If edge $(x, u)$ or $(x, v)$ exists in graph $G$, the edge $\left(x, v_{u, v}\right)$ is added to $\rho_{u, v}(G)$. Fig.3(a) shows the new graph after the vertices $v_{14}, v_{15}$ in Fig.1 are merged, and Fig.3(b) shows the new graph after the vertices $u_{1}, v_{12}$, and $v_{13}$ in Fig.3(a) are merged. 


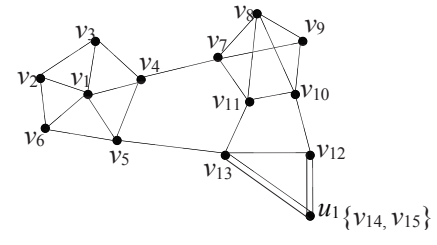

(a)

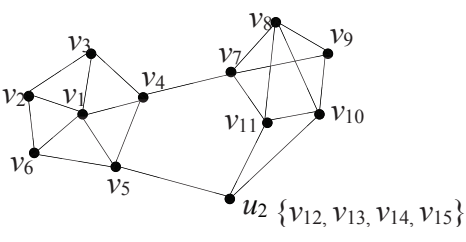

(b)

Fig. 3: The strategy of merging vertices. (a) Merging vertices $v_{14}$ and $v_{15}$ into a super-vertex $u_{1}$. (b) Merging vertices $u_{1}, v_{12}$ and $v_{13}$ into a super-vertex $u_{2}$

The proposed algorithm MSK iteratively applies early merging and splitting to compute $k$-edge connected components. For early splitting strategy, if a cut with value less than $k$ during the procedure of computing order list $L$, i.e. $w\left(V_{L}, V \backslash V_{L}\right)<k$, we decompose $G$ into two separate subgraphs $G[L]$ and $G[V \backslash L]$. For each iteration, we compute the order list $L$ of vertices of $G_{1}$ which is the subgraph of $P G$. If the cut with value $C=\left(S, V_{G_{1}} \backslash S\right)$ and $|C|<k$, graph $G_{1}$ is decomposed into two subgraphs by the cut $C$. During this decomposing procedure, the super-vertices in $V_{G_{1}} \backslash S$ are restored to the original state. Let $\operatorname{Org}\left(V_{G_{1}} \backslash S\right)$ denote the vertex set after restoring the super-vertices of $\left(V_{G_{1}} \backslash S\right)$ to the original state. If the subgraph of $P G$ is a super-vertex, all the original vertices constituting the super-vertex are returned. The subgraph of $G$ consist of the original vertices and their edges is a $k$-edge connected subgraph. The algorithm is terminated until $P G=\emptyset$. Since applying the vertices merging strategy, the proposed algorithm is convergent. However, for algorith$\mathrm{m}$ DecB-LMSD, after vertices merging, the new vertex is considered as a single super-vertex, which will make the result deviation. This is because during the processing of vertices merging, if vertex $t$ is inserted into $L$ and $w(L, t) \geq k$, the last vertex $s$ of $L$ and $t$ are merged, in the subsequent iteration, if the vertices in $L$ is divided into two disconnected subgraphs, the minimum cut value of merged vertices $s$ and $t$ might be smaller than $k$, therefore, the single super-vertex $V_{s, t}$ should be restored to the original state.

\subsection{Algorithm illustration}

Consider the graph $G$ in Fig.2, Fig.4 shows the procedure of finding $k$-edge connected components by using algorithm MSK when $k=3$. We compute an order list $L$ for the vertices of $G$, the information of list $L$ obtained from all iterations during the running of algorithm MSK is shown in Table 1.

Fig.4(a) shows the result after the first iteration. At first, $L=\emptyset$. The vertices traversal is started from $v_{1}$. Then, $v_{1}$ is added into $L$ and $L=\left\{v_{1}\right\}$. At 


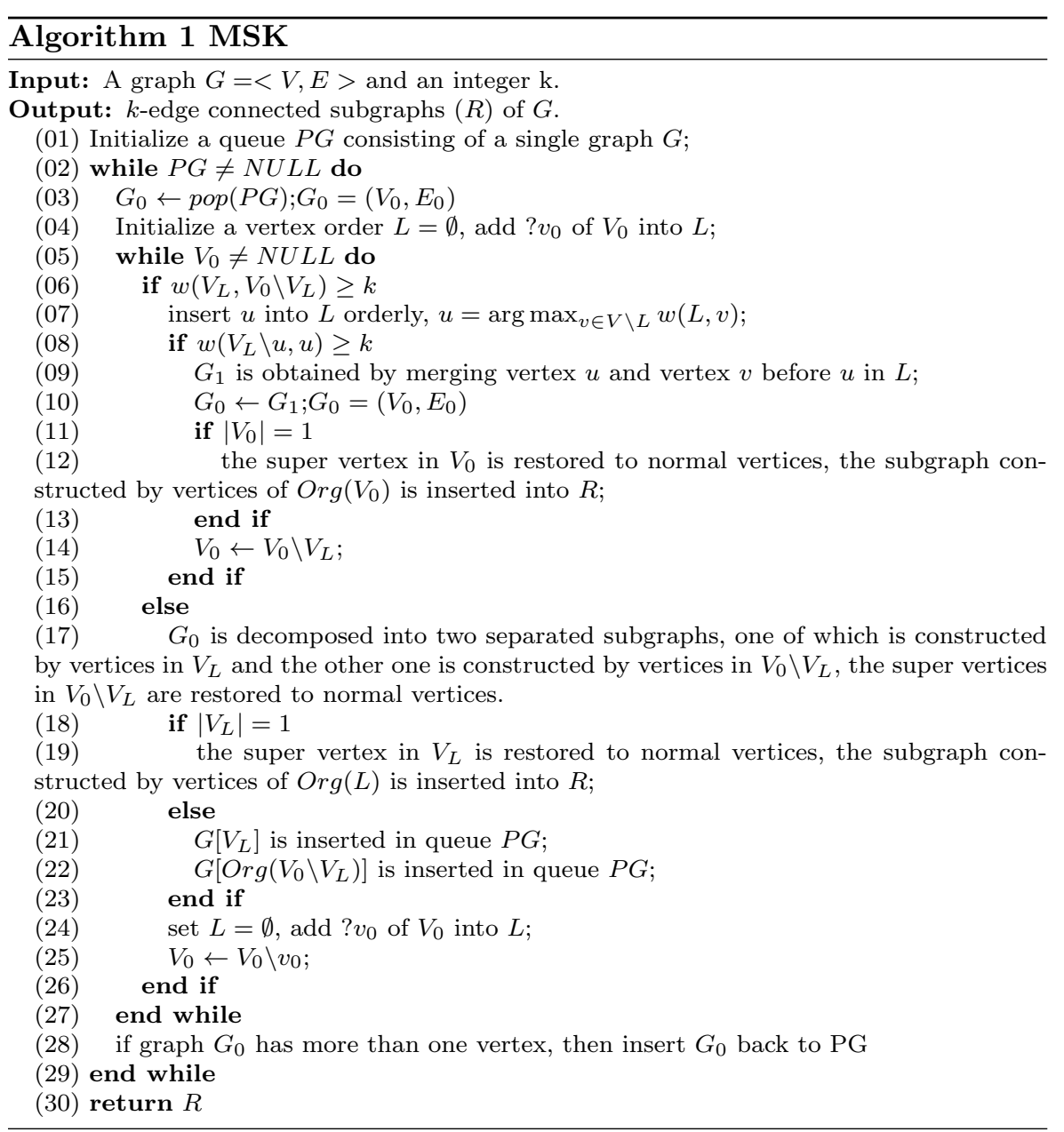

Table 1: Change of $L$ in the procedure of algorithm MSK on the sample graph $\tilde{G}$

\begin{tabular}{cc}
\hline Iteration & Order list $L$ \\
\hline 1 & $v_{1}, v_{2}, v_{3},\left\{v_{4}, v_{5}\right\}, v_{6}, v_{7}, v_{8},\left\{v_{9}, v_{10}\right\}, v_{11},\left\{v_{12}, v_{13}, v_{14}\right\}, v_{15},\left\{v_{16}, v_{17}\right\}$ \\
\hline 2 & $v_{1},\left\{v_{15}, v_{16}, v_{17}\right\}, v_{2}, v_{3}, v_{4}, v_{5}, v_{6}, v_{7}, v_{8},\left\{v_{9}, v_{10}\right\}, v_{11},\left\{v_{12}, v_{13}, v_{14}\right\}$ \\
\hline 3 & $\left\{v_{1}, v_{15}, v_{16}, v_{17}\right\},\left\{v_{4}, v_{11}, v_{12}, v_{13}, v_{14}\right\}, v_{5}, v_{6}, v_{7}, v_{8},\left\{v_{9}, v_{10}\right\}$ \\
\hline 4 & $v_{5},\left\{v_{6}, v_{9}, v_{10}\right\},\left\{v_{7}, v_{8}\right\}$ \\
\hline 5 & $v_{5},\left\{v_{6}, v_{7}, v_{8}, v_{9}, v_{10}\right\}$ \\
\hline 6 & $\left\{v_{5}, v_{6}, v_{7}, v_{8}, v_{9}, v_{10}\right\}$ \\
\hline
\end{tabular}




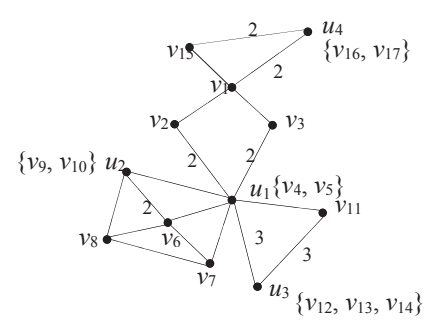

(a)

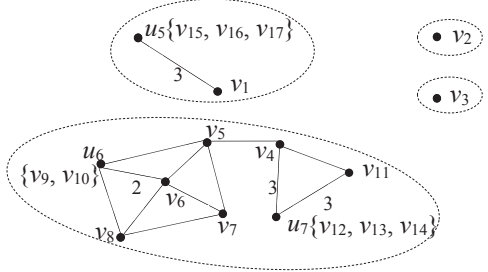

(c)

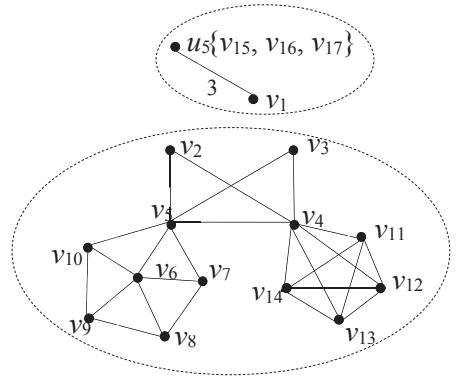

(b)

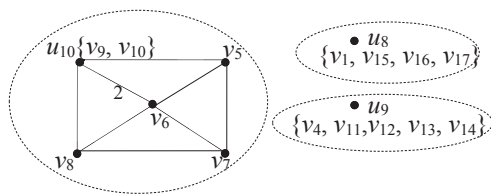

(d)

$$
\begin{aligned}
& \left\{v_{1}, v_{15}, v_{16}, v_{17}\right\} \\
& \left\{v_{4}, \stackrel{v}{11}_{11}, v_{12}, v_{13}, v_{14}\right\} \\
& \bullet u_{14} \\
& \left\{v_{5}, v_{6}, v_{7}, v_{8} v_{9}, v_{10}\right\}
\end{aligned}
$$

(e)

Fig. 4: The process of computing $k$-edge connected components by algorithm MSK. (a) Result graph after the first step of $L$. (b) Result graph after the second step of $L$. (c) Result graph after the third step of $L$. (d) The detected $k$-edge connected components

this time, the vertices $v_{2}, v_{3}, v_{15}, v_{16}$ and $v_{17}$ have the same connectivity degree with $L$, we select $v_{2}$ to be inserted into $L$. The connectivity degrees $w(v, L)$ of the remaining vertices are computed and the most closely connected vertices with $L$ is inserted into $L$ each time. When $L=\left\{v_{1}, v_{2}, v_{3}, v_{4}\right\}$, the most closely connected vertex is $v_{5}$. Since $w\left(v_{5}, L\right)=3 \geq k, v_{5}$ and the last vertex of $L$ are merged into a single super-vertex, which is denoted as $u_{1}$. The vertices connected with $v_{4}$ or $v_{5}$ in the original graph are connected with $u_{1}$. Since there exist edges $\left(v_{2}, v_{4}\right)$ and $\left(v_{2}, v_{5}\right)$ in the original graph $G$, the number of 
edges between $v_{2}$ and $u_{1}$ is equal to 2 , which can be denoted as $w\left(v_{2}, u_{1}\right)=2$. After the first iteration, we can get the result shown in Fig.4(a).

In the second iteration, the traversal is started from $v_{1}$, then, $L=\left\{v_{1}\right\}$. At this time, the super vertex $u_{4}$ composed of vertices $v_{16}$ and $v_{17}$ in Fig.4 (a) is most closely connected with $L$. After inserting $u_{4}$ into $L$, since $w\left(v_{15}, L\right)=$ $3 \geq k$, vertex $v_{15}$ is inserted into L. $v_{15}$ and $u_{4}$ are merged into a single super-vertex $u_{5}$. Since the number of edges between $L=v_{1}, u_{5}$ and $V \backslash L$ is 2, $w(L, V \backslash L)=2<k$, the graph is decomposed. Then, we get two subgraphs, one is composed by vertices $v_{1}$ and $u_{5}$, and another is composed by the remaining vertices. The graph decomposition may result in two k-edge connected vertices in the original graph do not satisfy the condition of $k$-edge connectivity in the subgraphs. Since the super-vertices inserted into $L$ satisfy the condition of $k$-edge connectivity in subgraph $G[L]$, only the super-vertices that are not inserted into $L$ need to be restored as the original state. Fig.4(b) shows the result after decomposition. The traversal of the remaining vertices is continued, the procedure is shown in Table 1 . Let $V=V \backslash L, L=\emptyset, v_{2}$ is inserted into $L$, since $w(L, V \backslash L)=2<k$, the split operation is executed again. Fig.4(c) shows the result after the second iteration, each ellipse region contains a subgraph. Since the subgraphs $\left\{v_{2}\right\}$ and $\left\{v_{3}\right\}$ only contain one vertex respectively, they are deleted from the graph. The other two subgraphs are processed as the input data during the next iteration.

In the third iteration, vertex $v_{1}$ is inserted into $L$ first, then the supervertex $u_{5}$ is inserted into $L$. At this time, we found that $v_{1}$ and $u_{5}$ are $k$-edge connected, they are merged into a super-vertex $u_{8}$. The $k$-edge connected subgraph composed of vertices $v_{1}, v_{15}, v_{16}$ and $v_{17}$ is obtained. Next, vertex $v_{4}$ is added into $L$ continuously. Since the vertex most closely connected with $v_{4}$ is $u_{7}$, then add $u_{7}$ into $L$. Next is $v_{11}$ which is most closely connected with $L$, the vertices $v_{11}, v_{4}$, and $u_{7}$ are merged into a super-vertex $u_{9}$. At this time, we found that the connectivity degree between $u_{9}$ and the remaining subgraphs is 1 which is less than $k$, the early splitting strategy is executed. Then, another subgraph composed of vertices $v_{4}, v_{11}, v_{12}, v_{13}$ and $v_{14}$ is obtained. Since the early splitting strategy is applied, the super-vertices that are not inserted into $L$ have to be restored as the original state. Fig.4(d) shows the three subgraphs after the third iteration. In the next iteration, the remaining vertices are processed, the subgraph composed of vertices $v_{5}, v_{6}, v_{7}, v_{8}, v_{9}$ and $v_{10}$ is obtained. Fig.4(e) shows the result after the final iteration, the vertex sets within the three ellipse regions corresponding to the three subgraphs $G_{1}, G_{2}$, and $G_{3}$ of Fig.2.

\subsection{Correctness analysis}

As for the correctness of vertices merging strategy, we can see that the minimum cut values among vertices which construct super-vertices should be not less than $k$. Given a connected graph $G=(V, E)$, let $s$ and $t$ be the last two vertices (ordered) in $L$, if $w(t, L \backslash t) \geq k$ and $\lambda(s, t ; G) \geq k$, then we can guarantee 
the correctness of merging vertices $s$ and $t$. However, if graph $G$ is decomposed into two subgraphs $G_{1}$ and $G_{2}$, it cannot guarantee that $\lambda(s, t ; G) \geq k$ in $G_{1}$ or $G_{2}$, which will result in an approximate result like DecB-LMSD algorithm. In order to resolve this problem, when graph $G$ has to be decomposed ( $w(L, V \backslash L)<k)$ in the proposed algorithm MSK, if $L$ contains super-vertex $u_{s, t}$, which indicate that $\lambda(s, t ; G(L)) \geq k$, the vertices in $L$ remain the same, the super-vertices in $V \backslash L$ has to be restored as the original state. This can guarantee that the minimum cut value among vertices of the subgraphs is not less than $k$ after each splitting. The correctness of vertices merging can be proved.

As for the correctness of early splitting strategy, we have to guarantee that a $k$-edge connected subgraph $g=\left(V_{g}, E_{g}\right)$ is not decomposed during the procedure of the graph $\mathrm{G}$ decomposing, i.e. the edges in $g$ cannot be removed. Since the next vertex which will be added into the order list $L$ is also a neighbor of the vertex contained in $L$, the graph $G$ can be early decomposed if the edge weight between the subgraph in $L$ and the remained subgraphs is less than $k$. Since the cut value of each splitting is less than $k$, the proving of the correctness of splitting strategy can be translated to prove the cut value that is less than $k$ cannot be the edges of $g$.

We prove it by contradiction. Assume that the cut $C=(S, T)$ contains edge $\{s, t\} \in E_{g}$, where $s \in S, t \in T$, and $|C|<k$. Since removing edges in $C$ will result in vertices $s$ and $t$ are disconnected, the minimum cut of $s-t$ is $\lambda(s, t ; G) \leq|C|$ and $\lambda(s, t ; g) \leq \lambda(s, t ; G)$, then $\lambda(s, t ; g)<k$. Since $g$ is $k$-edge connected subgraph, $\lambda(g) \geq k$, and $(s, t) \in E_{g}$. we can get that $\lambda(g) \leq$ $\lambda(s, t ; g)$, this means that $\lambda(s, t ; g) \geq k$, which contradicts that $\lambda(s, t ; g)<k$. The correctness of early splitting can be proved.

As for the correctness of returned results, since the correctness of vertices merging strategy and early splitting strategy, the vertices in each $k$-edge connected subgraph will be merged to a super vertex during the procedure of iterations. For each normal vertex set $V_{i}$ which constitute the super-vertices, $G\left(V_{i}\right)$ is a $k$-edge connected subgraph. This guarantee that the results are all of the $k$-edge connected subgraphs.

\subsection{Complexity analysis}

In this paper, the early merging and splitting based $k$-edge connected component detection algorithm MSK iteratively computes an order list of vertices named $L$ according to the connectivity of vertices in a graph to get the final results. The time complexity for each computation of order list $L$ is $O(|E|+|V| \log |V|)$ if Fibonacci heap is used to store the vertices list. The hash data structure proposed by DecB-LMSD can reduce the time complexity of order list $L$ computation to $O(|E|)$. The procedure of graph decomposition can be organized into a tree structure, each layer of the tree represents the result of each iteration. The $(i+1)$ th layer is obtained by merging and splitting the $i t h$ layer, which ensures that the result is convergent and the number 
of iterations is the height of the tree. Therefore, the total time complexity is $O(h \times|E|)$, where $h$ is the number of iterations and $|E|$ is the number of edges. The time complexity is bounded with the value of height $h$. The height $h$ of the decomposition tree reflects the depth of the $k$-edge connected components nested together to form the graph $G$. After each iteration of decomposition, the depth $h$ of nest is reduced by one.

The value of $h$ depends on several different factors. The more the number of $k$-edge connected components in a graph, the more likely we will get a higher $h$. If there exists $N k$-edge connected components in a graph, the height of the decomposition tree will be $N-1$ in the worst case. If these $k$-edge connected components are densely connected with each other and hard to decompose, then $h$ is potentially higher. The number of $k$-edge connected components largely relies on the parameter $k$. A higher $k$ leads to fewer $k$-edge connected components. Given a non $k$-edge connected graph, the more the number of disconnected subgraphs returned by each decomposition, the lower the height $h$ of the decomposition tree will be obtained. Usually, most of the $k$-edge connected components in the real graphs are sparsely connected with each other, which means that they can be split easily. Consequently, we will get more disconnected subgraphs, and we potentially get a smaller $h$. The low bound of $h$ is 1 , which indicates that the given input graph $G$ is $k$-edge connected and it does not need any decomposition.

Another factor that may affect the time efficiency of our algorithm is the choice of initial vertex. It changes the traverse order and might result in different $h$ and thus affect the time efficiency. For example, consider the graph in Fig.1, there are three $k$-edge connected components(here $k=3$ ) $G_{1}, G_{2}, G_{3}$ in it. The cut between $G_{1}, G_{2}, G_{3}$ and the rest part of the graph is 2,3 and 3 , respectively. If the traversal starts from $v_{1}$, the early merging and splitting strategy will first cut $G_{1}$ from the whole graph and then decompose the rest to $G_{2}$ and $G_{3}$. This can be done in one iteration, so $h$ equals 1 . However, if it starts from a node in $G_{2}$ or $G_{3}\left(\right.$ such as $v_{7}$ ), the algorithm only cut $G_{2} \cup G_{3}$ from the whole graph in the first iteration, and $G_{2}$ and $G_{3}$ will be split in the next iteration. So in this case $h$ is equal to 2 . In fact, according to the above analysis of $h$, the value of $h$ is usually very small, so even if the initial vertex selection will affect the size of $h$, the impact on the final computation complexity is very limited. This phenomenon is supported by our experiment studies.

\section{The proposed approximate algorithm PMSK}

The proposed algorithm MSK computing $k$-edge connected components by iteratively computing the order list $L$, and then get the final result. In order to obtain accurate result, the super vertex are restored as the original state during the iterations. However, this will slow down the processing speed of vertices. In order to improve the efficiency, we give an approximate algorithm 
Table 2: Contents of $L$ in the procedure of PMSK on the sample graph $\tilde{G}$

\begin{tabular}{cc}
\hline Iteration & Order list $L$ \\
\hline 1 & $v_{1}, v_{2}, v_{3},\left\{v_{4}, v_{5}\right\}, v_{6}, v_{7}, v_{8},\left\{v_{9}, v_{10}\right\}, v_{11},\left\{v_{12}, v_{13}, v_{14}\right\}, v_{15},\left\{v_{16}, v_{17}\right\}$ \\
\hline 2 & $v_{1},\left\{v_{15}, v_{16}, v_{17}\right\}, v_{2}, v_{3},\left\{v_{6}, v_{9}, v_{10}\right\},\left\{v_{4}, v_{5}, v_{11}, v_{12}, v_{13}, v_{14}\right\},\left\{v_{7}, v_{8}\right\}$ \\
\hline 3 & $\left\{v_{1}, v_{15}, v_{16}, v_{17}\right\},\left\{v_{4}, v_{5}, v_{6}, v_{7}, v_{8}, v_{9}, v_{10}, v_{11}, v_{12}, v_{13}, v_{14}\right\}$ \\
\hline
\end{tabular}

Table 3: Contents of $L$ in the procedure of DecB-LMSD on the sample graph $G$

\begin{tabular}{cc}
\hline Iteration & Order list $L$ \\
\hline 1 & $v_{1}, v_{2}, v_{3},\left\{v_{4}, v_{5}\right\}, v_{6}, v_{7}, v_{8},\left\{v_{9}, v_{10}\right\}, v_{11},\left\{v_{12}, v_{13}, v_{14}\right\}, v_{15},\left\{v_{16}, v_{17}\right\}$ \\
\hline 2 & $v_{1},\left\{v_{15}, v_{16}, v_{17}\right\}, v_{2},\left\{v_{6}, v_{9}, v_{10}\right\},\left\{v_{7}, v_{8}\right\},\left\{v_{3}, v_{4}, v_{5}, v_{11}, v_{12}, v_{13}, v_{14}\right\}$ \\
\hline 3 & $\left\{v_{1}, v_{15}, v_{16}, v_{17}\right\}, v_{2},\left\{v_{3}, v_{4}, v_{5}, v_{6}, v_{7}, v_{8}, v_{9}, v_{10}, v_{11}, v_{12}, v_{13}, v_{14}\right\}$ \\
\hline 4 & $\left\{v_{1}, v_{15}, v_{16}, v_{17}\right\},\left\{v_{12}, v_{3}, v_{4}, v_{5}, v_{6}, v_{7}, v_{8}, v_{9}, v_{10}, v_{11}, v_{12}, v_{13}, v_{14}\right\}$ \\
\hline 5 & $\left\{v_{1}, v_{15}, v_{16}, v_{17}\right\},\left\{v_{12}, v_{3}, v_{4}, v_{5}, v_{6}, v_{7}, v_{8}, v_{9}, v_{10}, v_{11}, v_{12}, v_{13}, v_{14}\right\}$ \\
\hline
\end{tabular}

PMSK, which ignores the restoring of super-vertices during the processing of vertices.

The proposed approximate algorithm PMSK also iteratively applies early merging and splitting to compute $k$-edge connected components. We use $P G$ to indicate the set of subgraphs generated by merging or splitting for the process of next iteration. For each iteration, we compute the order list $L$ of vertices of $G_{1}$ which is the subgraph of $P G$. If a cut with value less than $k$ during the procedure of computing order list $L$, i.e. $w\left(V_{L}, V \backslash V_{L}\right)<k$, we decompose $G$ into two separate subgraphs $G[L]$ and $G[V \backslash L]$. During this decomposing procedure, if the vertices are $k$-edge connected, they are merged into a single super-vertex. The original vertices and their related edges are deleted from graph $G$. The algorithm is terminated until $P G=\emptyset$.

Although DecB-LMSD can also be considered as an approximate approach, the proposed algorithm PMSK is more efficient than it and the result is closer to the accurate result. Table 2 and Table 3 show the procedure of computing $k$ edge connected subgraphs for the graph G in Fig.2 by using PMSK and DecBLMSD respectively. In Table 2, the graph $G$ is decomposed into four subgraphs after the second iteration of computing $L$ by using algorithm PMSK. Since the vertices $v_{2}$ and $v_{3}$ are isolated, they are removed from $L$. The other two subgraphs are proposed in the next iteration. The final result can be computed after the third iteration. In Table 3, for algorithm DecB-LMSD, the graph $G$ is decomposed when the minimum cut value of the last two vertices of $L$ is less than $k$. The graph $G$ is decomposed after the fifth iteration. From the results we can see that the vertices that do not satisfy $k$-edge connected condition are eliminated during the early splitting operation by algorithm PMSK, such as vertices $v_{2}$ and $v_{3}$. However, these unnecessary vertices cannot be filtered out by algorithm DecB-LMSD. Therefore, the result of algorithm PMSK is closer to the accurate result. 
Table 4: The information of the compared algorithms

\begin{tabular}{c|ccc}
\hline Algorithm & Exact & Approximate & Description \\
\hline CutB-TC & $\checkmark$ & & The cut-based framework + TestConnect algorithm \\
\hline MSK & $\checkmark$ & & Our accurate method using decomposition-based framework \\
\hline DecB-LMSD & & $\checkmark$ & The decomposition-based framework + graph decomposition algorithm \\
\hline PMSK & & $\checkmark$ & Our approximate method using decomposition-based framework \\
\hline
\end{tabular}

\section{Experimental results and analysis}

In this section, we conduct extensive performance experiments to evaluate the efficiency of the proposed method and compare it with existing methods. All experiments are conducted on a desktop PC with a $3.20 \mathrm{GHz} \mathrm{CPU}$ and 4GB memory. All of the algorithms are implemented in ANSI C++. The proposed graph decomposition algorithm applies merging and splitting strategy to find $k$-edge connected subgraphs. As shown in Table 4, algorithm MSK can compute the accurate result and PMSK is an approximate algorithm. We compare the proposed algorithms with the other existing graph decomposition algorithms DecB-LMSD and cut-based exact algorithm CutB-TC.

\subsection{Datasets}

We evaluate the performance of all the algorithms on both real and synthetic graphs. The synthetic graphs are generated by the graph generator developed by Lancichinetti et al ${ }^{1}$. We generate eight synthetic graphs with the number of 1,000 vertices and eight synthetic graphs with the number of 10,000 vertices, respectively. The number of edges is 20 times of that of vertices in each graph. We also evaluate the algorithms on eight real graphs, such as Political Blogs network (Political Blogs), Arxiv General Relativity collaboration network (caGrQc), the graph of routers comprising the Internet can be organized into subgraphs called Autonomous Systems (as-735), Arxiv HEP-TH (High Energy Physics - Theory) collaboration network (ca-HepTh), Arxiv Condensed Matter collaboration network (ca-CondMat), author collaboration network in DBLP (DBLP Co-authorship), Arxiv HEP-TH (high energy physics theory) citation graph (cit-HepPh), and who-trusts-whom network of Epinions.com (soc-Epinions), All the graphs are downloaded from the Stanford SNAP library ${ }^{2}$, and detailed descriptions about these graphs can also be found there. The sizes of these graphs are shown in Table 5.

\subsection{Accuracy evaluation}

Table 6 shows the results of each algorithm on the real graphs. The number of subgraphs denotes the number of $k$-edge connected components computed by

\footnotetext{
1 A software package to generate the benchmark graphs can be downloaded from http://santo.fortunato.googlepages.com/benchmark.tgz

2 http://snap.stanford.edu/data/
} 
Table 5: The sizes of the real graphs

\begin{tabular}{c|cc}
\hline Dataset & \#Vertices & \#Edges \\
\hline Political Blogs & 1,490 & 19,090 \\
\hline ca-GrQc & 5,242 & 14,496 \\
\hline as-735 & 6,475 & 12,572 \\
\hline ca-HepTh & 9,877 & 51,971 \\
\hline ca-CondMat & 23,133 & 93,497 \\
\hline DBLP & 28,702 & 66,832 \\
\hline cit-HepPh & 34,546 & 421,578 \\
\hline soc-Epinions1 & 75,875 & 508,837 \\
\hline
\end{tabular}

Table 6: $k$-edge connected subgraphs detected in real graphs by different algorithms

\begin{tabular}{c|c|cccc}
\hline Real graphs & & MSK & CutB-TC & PMSK & DecB-LMSD \\
\hline Political Blogs & Number of components & 1 & 1 & 1 & 1 \\
& Max size of components & 894 & 894 & 984 & 1,208 \\
\hline ca-GrQc & Number of components & 1 & 1 & 1 & 1 \\
& Max size of components & 1,350 & 1,350 & 1,349 & 1,368 \\
\hline as-735 & Number of components & 1 & 1 & 1 & 1 \\
& Max size of components & 485 & 485 & 485 & 485 \\
\hline ca-HepTh & Number of components & 32 & 32 & 32 & 32 \\
& Max size of components & 3,068 & 3,068 & 3,581 & 3,071 \\
\hline ca-CondMat & Number of components & 83 & 83 & 85 & 85 \\
& Max size of components & 12,917 & 12,917 & 12,922 & 12,923 \\
\hline DBLP & Number of components & 296 & 296 & 309 & 309 \\
& Max size of components & 7,342 & 7,342 & 7,338 & 7,338 \\
\hline cit-HepPh & Number of components & 2 & 2 & 3 & 3 \\
& Max size of components & 30,196 & 30,196 & 30,196 & 30,196 \\
\hline soc-Epinions1 & Number of components & 9 & 9 & 11 & 11 \\
& Max size of components & 19,581 & 19,581 & 19,581 & 19,581 \\
\hline
\end{tabular}

each algorithm, and the max size of subgraphs denotes the subgraph with the maximum number of vertices within the generated $k$-edge connected subgraphs by graph decomposition algorithms. The differences between the number of $k$ edge connected subgraphs and the max size of subgraph for PMSK and DecBLMSD are not obvious. The algorithm MSK and algorithm CutB-TC have the same number of $k$-edge connected subgraphs and the max size of subgraph. This is because both MSK and CutB-TC can compute the accurate results and the results of PMSK and DecB-LMSD are approximate.

Table 7 shows the results change of the proposed method when varying the input parameter $k$. With the increase of the parameter $k$, the number of subgraphs and the max size of subgraphs are decreased. When the value of $k$ reaches a certain level, the satisfied subgraphs are not exist.

In conclusion, comparing with the exact algorithm CutB-TC, our exact algorithm MSK can also find the ground truth of the $k$-edge connected components in a graph. The proposed approximate algorithm PMSK possesses not only better time efficiency but also more accurate results than the other approximate method DecB-LMSD. 
Table 7: $k$-edge connected subgraphs detected in real graphs by different algorithms with varying parameter $k$

\begin{tabular}{c|l|cccccc}
\hline Real graphs & & $k=2$ & $k=3$ & $k=4$ & $k=5$ & $k=10$ & $k=20$ \\
\hline Political Blogs & Number of components & 2 & 1 & 1 & 1 & 1 & 1 \\
& Max size of components & 1,081 & 894 & 894 & 849 & 676 & 425 \\
\hline ca-GrQc & Number of components & 168 & 65 & 30 & 18 & 10 & 0 \\
& Max size of components & 3,240 & 2,248 & 1,350 & 745 & 80 & 0 \\
\hline as-735 & Number of components & 1 & 1 & 1 & 1 & 1 & 0 \\
& Max size of components & 4,023 & 1,301 & 485 & 240 & 36 & 0 \\
\hline ca-HepTh & Number of components & 185 & 81 & 32 & 16 & 3 & 0 \\
& Max size of components & 6,818 & 4,683 & 3,068 & 1,992 & 32 & 0 \\
\hline ca-CondMat & Number of components & 301 & 160 & 83 & 48 & 13 & 2 \\
& Max size of components & 19,378 & 16,135 & 12,917 & 10,002 & 2,083 & 28 \\
\hline DBLP & Number of components & 1,276 & 657 & 296 & 231 & 40 & 12 \\
& Max size of components & 17,161 & 12,678 & 7,342 & 6,345 & 1,785 & 268 \\
\hline cit-HepPh & Number of components & 18 & 4 & 2 & 1 & 1 & 1 \\
& Max size of components & 33,072 & 31,720 & 30,196 & 28,603 & 20,597 & 7,667 \\
\hline
\end{tabular}

\subsection{Processing time evaluation}

We evaluate the algorithms on two kinds of synthetic graphs, a graph set contains eight graphs with 1,000 vertices and the other graph set contains eight graphs with 10000 vertices. Since the degree of the generated graphs is 40 , in order to avoid the generated graph is $k$-edge connected the integer $k$ is set to 10. Fig. 5 shows the results of MSK, PMSK and DecB-LMSD on these two graph sets. Since the running time of CutB-TC is much longer than that of the other algorithms, it is omitted from Fig.5(a) and Fig.5(b). The processing time is CPU time. The processing time of algorithm MSK is a little longer than that of the other algorithms sometimes, but it can compute accurate results. Both algorithm PMSK and DecB-LMSD can compute approximate results, but the efficiency of PMSK algorithm is better than that of DecB-LMSD. In order to compare the efficiency of the proposed methods with existing methods, we generate eight synthetic graphs with 5,000 vertices and the number of edges is from 15,000 to 32,500 . The input parameter $k$ is set to 9 . Fig. 6 shows that, the proposed algorithm MSK is fast when the density is low, the running times reach a steady value when the density is high enough. The algorithm PMSK is a little faster than the other algorithms.

Since the connectivity of real graph is not high, for each real graph we select $k=4$ when performing MSK algorithm, PMSK algorithm, DecB-LMSD algorithm, and CutB-TC algorithm to compute $k$-edge connected subgraphs. Table 8 shows the time consumptions of each algorithm on real graphs. From the results, we can see that the time efficiency of algorithm MSK outperforms algorithm CutB-TC and the time efficiency of algorithm PMSK outperforms algorithm DecB-LMSD.

Table 9 shows the running times of the proposed method when varying the input parameter $k$. With the increase of the parameter $k$, the running time is 


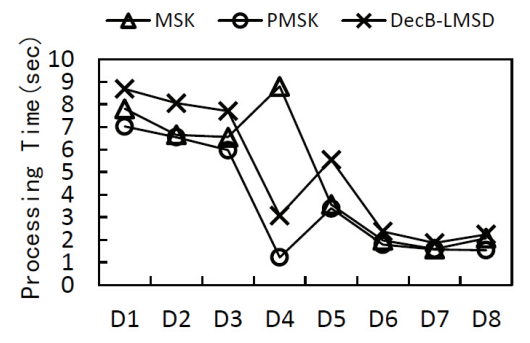

(a)

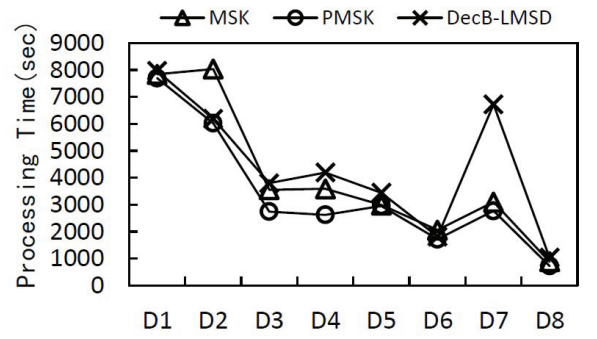

(b)

Fig. 5: Processing times of the three algorithms on synthetic graphs. (a) Graphs with 1,000 vertices. (b) Graphs with 10,000 vertices

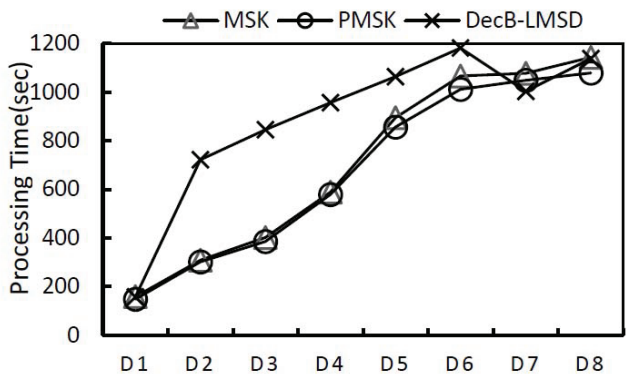

Fig. 6: Processing time with different densities of the graphs

Table 8: Processing time (Sec.) on real graphs

\begin{tabular}{c|cccc}
\hline Real graphs & MSK & CutB-TC & PMSK & DecB-LMSD \\
\hline Political Blogs & 0.28 & 2.56 & 0.18 & 0.31 \\
\hline ca-GrQc & 23.57 & 76.54 & 21.75 & 40.07 \\
\hline as-735 & 303.55 & 573.11 & 267.22 & 432.13 \\
\hline ca-HepTh & 195.72 & 643.72 & 189.73 & 348.432 \\
\hline ca-CondMat & 895.78 & $1,785.64$ & 871.38 & $1,176.16$ \\
\hline DBLP & $2,671.86$ & $5,879.23$ & $1,960.08$ & $3,046.63$ \\
\hline cit-HepPh & 563.75 & $1,374.94$ & 527.17 & 651.12 \\
\hline soc-Epinions1 & $1,629.15$ & $5,843.72$ & $1,408.70$ & $3,046.63$
\end{tabular}

increased. When the value of $k$ reaches a certain level, the satisfied subgraphs are not exist and the running time is decreased obviously.

In conclusion, compared with the exact algorithm CutB-TC, the proposed exact algorithm MSK is more efficient than it. And the algorithm PMSK has better time efficiency than the other approximate algorithm DecB-LMSD. 
Table 9: Processing time (Sec.) with different values of parameter $k$

\begin{tabular}{c|cccccc}
\hline Real graphs & $k=2$ & $k=3$ & $k=4$ & $k=5$ & $k=10$ & $k=20$ \\
\hline Political Blogs & 0.184 & 0.229 & 0.281 & 0.275 & 0.385 & 0.79 \\
\hline ca-GrQc & 23.206 & 18.842 & 23.57 & 43.104 & 0.596 & 0.473 \\
\hline as-735 & 51.834 & 288.967 & 303.55 & 303.92 & 167.867 & 83.1 \\
\hline ca-HepTh & 102.401 & 145.222 & 195.72 & 348.461 & 467.162 & 0.51 \\
\hline ca-CondMat & 471.764 & 640.528 & 895.78 & $2,244.31$ & $9,687.15$ & $7,055.96$ \\
\hline DBLP & $2,613.058$ & $2,388.965$ & $2,671.86$ & $2,612.863$ & $3,187.093$ & $2,149.612$ \\
\hline cit-HepPh & 69.643 & 93.181 & 563.75 & 420.017 & $6,338.384$ & $76,389.562$ \\
\hline
\end{tabular}

Table 10: Value of $h$ of $\operatorname{PMSK}(k=10)$

\begin{tabular}{c|ccc}
\hline Real graphs & Number of components & Max Size of components & $h$ \\
\hline Political Blogs & 1 & 676 & 2 \\
\hline ca-GrQc & 10 & 80 & 6 \\
\hline as-735 & 1 & 36 & 2 \\
\hline ca-HepTh & 3 & 32 & 2 \\
\hline ca-CondMat & 13 & 2083 & 20 \\
\hline DBLP & 40 & 1785 & 14 \\
\hline cit-HepPh & 1 & 20597 & 6 \\
\hline
\end{tabular}

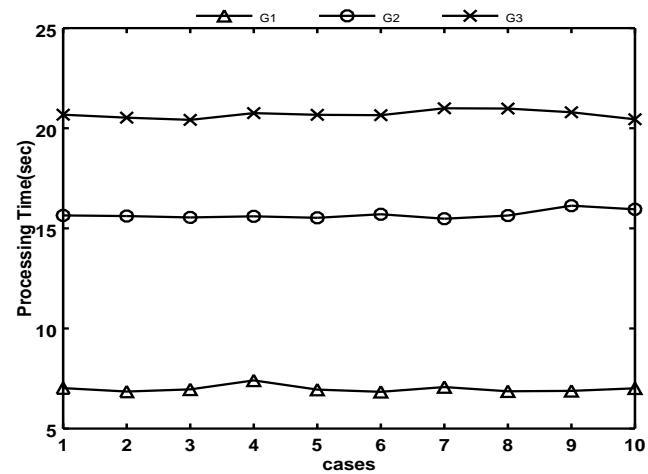

Fig. 7: Processing time in different case of initial vertex

6.4 Evaluation of the decomposition tree height $h$ and the choice of initial vertex

The value of $h$ contributes to the time complexity $O(h \times|E|)$ of our algorithm. Table 10 shows the value of $h$ by applying PMSK on seven real graphs as well as the number and the max size of $k$-edge connected components with $k=10$. Among these real graphs, ca-CondMat, DBLP and cit-HepPh have the largest number of $k$-edge connected components and their max size of components are also very large. In spite of the large number of $k$-edge connected components and the large size of $k$-edge connected components, the value of $h$ in PMSK algorithm is small. 
To evaluate how the choice of initial vertex affect the time efficiency of our algorithm, we run PMSK on three synthetic graphs under different cases of initial vertex with $k=10$. Fig. 7 shows the processing time of PMSK in different cases of initial vertex on these graphs. The average degree of these generated graphs is 15 and the node numbers of G1, G2 and G3 are 3000, 5000 and 7000 , respectively. For each graph, 10 different copies of the original graph are obtained by randomly relabeling it. Then, we get the running time of PMSK under different choices of initial vertex by starting from the specified node label in each copy. We run each experiment three times, and report the average CPU time. Fig.7 shows that PMSK's running time vary with the choices of initial vertex but keep very close with each other.

\section{Conclusion}

In this paper, we propose an algorithm MSK to compute $k$-edge connected components. The proposed method iteratively computes an order list $L$ based on the connectivity of vertices within a graph $G$. In order to improve the efficiency, we propose early merging and early splitting strategies. During the computing process of the order list $L$, if two vertices are $k$-edge connected, the merging operation is performed and the two $k$-edge connected vertices are merged into a super-vertex. If the cut value between two vertices is less than $k$, the splitting operation is preformed and the graph is decomposed by removing all edges with cut value that less than $k$. The above procedure is performed iteratively to compute $k$-edge connected subgraphs. The experimental results on a large number of real-world and synthetic graphs show that the proposed algorithm is accurate and is more efficient than the state-of-the-art methods.

\section{Acknowledgment}

The work was supported in part by the National Science Foundation of China grants 61173093, 61202182 and 61474299, the Fundamental Research Funds for the Central Universities of China grants K5051323001 and BDY10, Shaanxi Postdoctoral Science Foundation, Natural Science Basic Research Plan in Shaanxi Province of China grants 2014JQ8359. Any opinions, findings, and conclusions expressed here are those of the authors and do not necessarily reflect the views of the funding agencies.

\section{References}

1. Chang L, Yu JX, Qin L, Lin X, Liu C, Liang W (2013) Efficiently computing $k$-edge connected components via graph decomposition. In: Proceedings of the 2013 ACM international conference on management of data, SIGMOD 2013, ACM, New York, pp205-216.

2. Zhang Y, Parthasarathy S (2012)Extracting analyzing and visualizing triangle $k$-core motifs within networks. In: Proceedings of the IEEE 28th international conference on data engineering, ICDE 2012, IEEE computer society, Washington, pp1049-1060. 
3. Fung WS, Hariharan R, Harvey NJA, Nicholas J (2011) A general framework for graph sparsification. In: Proceedings of the 43rd ACM symposium on theory of computing, STOC 2011, ACM, San Jose, pp71-80.

4. Wang N, Zhang J, Tan KL, Tung AK (2010) On triangulation-based dense neighborhood graph discovery. Proc. VLDB Endowment. 4(2):58-68.

5. Agrawal R, Rajagopalan S, Srikant R, Xu Y (2003) Mining newsgroups using networks arising from social behavior. In: Proceedings of the 12th international conference on World Wide Web, WWW 2003, ACM, New York, pp529-535.

6. Bencz A, Karger DR (2015) Randomized approximation schemes for cuts and flows in capacitated graphs. SIAM J. Comput. 44(2):290-319.

7. Stoer M, Wagner F (1997) A simple min-cut algorithm. Journal of the ACM, 44(4):585591.

8. Yan X, Zhou XJ, Han J (2005) Mining closed relational graphs with connectivity constraints. In: Proceedings of the eleventh ACM SIGKDD international conference on knowledge discovery and data mining, KDD 2005, ACM, New York, pp324-333.

9. Zhou R, Liu C, Yu JX, Liang W, Chen B, Li J (2012) Finding maximal k-edge-connected subgraphs from a large graph. In: Proceedings of the 15th international conference on extending database technology, EDBT 2012, ACM, New York, pp480-491.

10. Ding C, He X, Zhang H, Gu M, Simon HD (2001) A min-max cut algorithm for graph partitioning and data clustering. In: Proceedings of the 2001 IEEE international conference on data mining, ICDM 2001, IEEE computer society, pp107-114.

11. Satuluri V, Parthasarathy S, Ruan Y (2011) Local graph sarsification for scalable clustering. In: Proceedings of the 2011 ACM SIGMOD international conference on management of data, SIGMOD 2011, ACM, New York, pp721-732.

12. Cheng J, Ke Y, Chu S, Ozsu MT (2011) Effcient core decomposition in massive networks. In: Proceedings of the IEEE 27th international conference on data engineering, ICDE 2011, IEEE computer society, pp51-62.

13. Gunnemann S, Boden B, Seidl T (2012) Finding density-based subspace clusters in graphs with feature vectors. Data Mining and Knowledge Discovery. 25(2): 243-269.

14. Chartrand G (1966) A graph-theoretic approach to a communications problem. SIAM J. Appl. Math. 14(4):778-781.

15. Karypis G, Kumar V (1998) A fast and high quality multilevel scheme for partitioning irregular graphs. SIAM Journal on Scientific Computing, 20(1):359-392

16. Goldberg AV, Tarjan RE (1988) A new approach to the maximum-flow problem. Journal of the ACM, 35(4):921-940.

17. Dankelmann P, Volkmann L (1997) Degree sequence conditions for maximally edgeconnected graphs and digraphs. Journal of Graph Theory, 26(1): 27-34.

18. Blum A, Chawla S (2001) Learning from labeled and unlabeled data using graph mincuts. In: Proceedings of the 18th international conference on machine learning, ICML 2001, Morgan Kaufmann, pp19-26.

19. Raghavan UN, Albert R, Kumara S. (2007) Near Linear Time Algorithm to Detect Community Structures in Larges Calenetworks. Phys. Rev. E, 76:036106.

20. Akiba T, Iwata Y, Yoshida Y (2013) Linear-time enumeration of maximal K-edgeconnected subgraphs in large networks by random contraction. In: Proceedings of the 22nd ACM international conference on information and knowledge management, CIKM 2013, ACM, pp909-918.

21. Li X, Neumann-Lara V, Rivera-Campo E (2005) Two approaches for the generalization of leaf edge exchange graphs on spanning trees to connected spanning k-edge subgraphs of a graph. Ars Comb., 75.

22. Jordan $\mathrm{T}$ (2005) On the existence of k edge-disjoint 2-connected spanning subgraphs. Journal of Combinatorial Theory Series B, 95(2):257-262. 